\title{
A Criterion for the Similarity of Length-Two Elements in a Noncommutative PID ${ }^{1)}$
}

\author{
Ziming Li Huaifu Wang ${ }^{2)}$ \\ Key Laboratory of Mathematics Mechanization \\ Institute of Systems Science, AMSS, Academia Sinica \\ Beijing 100190, China \\ \{zmli,wanghf\}@mmrc.iss.ac.cn
}

\begin{abstract}
We present a criterion for the similarity of length-two elements in a noncommutative principal ideal domain. The criterion enables us to develop an algorithm for determining whether $B_{1} A_{1}$ and $B_{2} A_{2}$ are similar, where $A_{1}, A_{2}, B_{1}, B_{2}$ are first-order differential (difference) operators. The main step in the algorithm is to find a rational solution of a parametric differential (difference) Risch's equation, which has been well-studied in symbolic integration (summation).
\end{abstract}

\section{Introduction}

Similarity is an equivalence relation among ordinary differential (difference) operators. This notion was extensively used by $\mathrm{O}$. Ore in [7] to describe the uniqueness of factorization of differential (difference) operators. Moreover, two differential (difference) operators are similar if and only if there exists a one-to-one correspondence, given by a differential (difference) operator, between their solution spaces. When the solution space of an operator is determined, so are the solution spaces of the similar ones (see [4]).

A general method for determining similarity is as follows. Let $L_{1}$ and $L_{2}$ be two differential operators of the same order over a differential field $k$. Then $L_{1}$ and $L_{2}$ are similar if and only if there exists a nonzero differential operator $U$ over $k$ such that $L_{1} U$ is right divisible by $L_{2}$, and that $U$ and $L_{2}$ have a trivial greatest common right divisor. The operator $U$ is called a solution of the mixed equation

$$
L_{1} Z \equiv 0 \quad \bmod L_{2} .
$$

Some applications of differential mixed equations are given in [10]. An efficient method for solving differential mixed equations is developed in [4].

Suppose that $L_{1}$ and $L_{2}$ are given as products of irreducible factors. It is naturally expected that one can use their factors to determine whether $L_{1}$ and $L_{2}$ are similar. In this paper, we present a criterion for the similarity of length-two elements in a noncommutative principal ideal domain (abbreviated as PID). Note that a ring of differential (difference) operators is a special instance for PID's. As an application, we develop an algorithm for

1) This work is sponsored in part by a 973 key project (no. 2004CB318000) and an NSFC project (no. $76596100)$

${ }^{2)}$ Corresponding author. 
determining whether $B_{1} A_{1}$ and $B_{2} A_{2}$ are similar, where $A_{1}, A_{2}, B_{1}, B_{2}$ are first-order differential (difference) operators. Proposition 5.4, the main result in Section 5., may be viewed as a generalization of the criterion for the similarity of degree-one Ore polynomials in $[6$, page 15]. The input operators $L_{1}$ and $L_{2}$ are not in factored form in van Hoeij's algorithm. So one would have to expand these two operators to apply the algorithm directly.

The rest of this paper is organized as follows. In Section 2., we present some basic results about noncommutative PID's that will be used later. In Section 3., we study how to determine the similarity of completely reducible elements using their irreducible decompositions. The results in Sections 2. and 3. are well-known, but their proofs are scattered in $[5,6,7]$. So we prove some of the results for a later convenience of reference. Section 4. contains a criterion for the similarity of non-completely-reducible elements with length two (Proposition 4.2); while its applications to Ore polynomials are given in Section 5.. We present a few examples in Section 6., and discuss on future work in Section 7..

\section{Preliminaries}

A PID is a (noncommutative) ring with a multiplicative unit 1 and without zero divisors, in which all one-sided ideals are principal. Typical examples are the rings of ordinary differential and difference operators, and univariate Ore polynomial rings over a field.

Through this paper, $R$ is a PID. For an element $a$ of $R, R a$ stands for the left ideal generated by $a$. We shall work with left ideals $R a$ and the corresponding factor $R / R a$, which is a cyclic left module over $R$ with a generator $1+R a$. By symmetry, the results will apply equally well to right ideals. Two nonzero elements $a$ and $b$ in $R$ are said to be similar if the left $R$-modules $R / R a$ and $R / R b$ are isomorphic. We denote by $a \sim b$ if $a$ and $b$ are similar. The similarity is an equivalence relation.

A remark is needed about homomorphisms between $R / R a$ and $R / R b$. For an element $c$ in $R$, the right-hand multiplication by $c$ gives rise to a left-module homomorphism:

$$
\begin{aligned}
\phi_{c}: \quad R / R a & \rightarrow R / R b \\
r+R a & \mapsto r c+R b .
\end{aligned}
$$

if and only if $R(a c) \subset R c \cap R b$. Now, let $\psi$ be a homomorphism from $R / R a$ to $R / R b$ with $\psi(1+R a)=c+R b$. Then $\psi=\phi_{c}$, because $R / R a$ is generated by $1+R a$ over $R$. In other words, every left module homomorphism from $R / R a$ to $R / R b$ is given by a righthand multiplication. This observation allows us to say that a left module homomorphism $\psi$ from $R / R a$ to $R / R b$ is given by $1+R a \mapsto c+R b$. Assume further that $a, b, c$ are nonzero elements of $R$, and that $\phi_{c}$ given above is well-defined. It is straightforward to verify that $\phi_{c}$ is surjective if and only if $R b+R c=R$; and that $\phi_{c}$ is injective if and only if $R(a c)=R b \cap R c$.

The next lemma is an ideal-theoretic characterization on similarity of elements, which is derived from [5, page 33].

Lemma 2.1 Let $R$ be a PID. For two nonzero elements $a, b \in R, a \sim b$ if and only if there exists a nonzero element $c \in R$ such that

$$
R(a c)=R b \cap R c \text { and } R b+R c=R .
$$


Moreover, the element $c$ in the two equalities in (1) can be replaced by any element that is congruent to $c$ modulo Rb.

Proof. By the above remark, $a \sim b$ if and only if (1) holds for some $c \in R$. The last assertion follows from the observation that

$$
1 \mapsto c+R b \quad \text { and } \quad 1 \mapsto \hat{c}+R b
$$

define the same homomorphism, provided that $c-\hat{c}$ is in $R b$.

For nonzero elements $a_{1}, \ldots, a_{n}$ in a PID $R$, there exist $d$ and $m$ in $R$ such that

$$
R a_{1}+\cdots+R a_{n}=R d \quad \text { and } \quad R a_{1} \cap \cdots \cap R a_{n}=R m .
$$

Note that $d$ and $m$ are nonzero and unique up to a unit left factor, respectively (see [5, page $31]$ ). We call $d$ a greatest common right-hand divisor (abbreviated as gcrd) of $a_{1}, \ldots, a_{n}$, and $m$ a least common left-hand multiple (abbreviated as $1 \mathrm{clm}$ ) of $a_{1}, \ldots, a_{n}$.

A nonzero element $a$ of $R$ is said to be irreducible if it is not a unit and has no proper factors, or, equivalently, the left ideal $R a$ is maximal in $R$. The following lemma describes a fundamental property of PID's (see [5, Chapter 3, Theorem 5]).

Lemma 2.2 Let $R$ be a PID. Then any nonzero nonunit element $a \in R$ can be written as a product of irreducible elements. If $a=p_{1} \cdots p_{n}=q_{1} \cdots q_{\ell}$, where the $p_{i}$ and $q_{j}$ are irreducible, then $n=\ell$, and the $p_{i}$ and the $q_{j}$ may be paired into similar pairs.

The number $n$ of irreducible factors $p_{i}$ in $a=p_{1} \cdots p_{n}$ is called the length of $a$ and denoted by length $(a)$. The length of a unit in $R$ is set to be zero. Then

$$
\text { length }(a b)=\text { length }(a)+\text { length }(b)=\text { length }(d)+\text { length }(m)
$$

for all nonzero $a, b \in R$, where $d$ and $m$ are a gcrd and an lclm of $a$ and $b$, respectively (see [5, Chapter 3, Theorem 6] and [6, Chapter 1, Proposition 1.2.11] ). The above irreducible decomposition of $a$ induces a composition series

$$
0 \subset R\left(p_{2} \cdots p_{n}\right) / R a \subset \cdots \subset R\left(p_{n-1} p_{n}\right) / R a \subset R p_{n} / R a \subset R / R a .
$$

The factors of this series are

$$
R\left(p_{2} \cdots p_{n}\right) / R a, R\left(p_{3} \cdots p_{n}\right) / R\left(p_{2} p_{3} \cdots p_{n}\right), \ldots, R\left(p_{n}\right) / R\left(p_{n-1} p_{n}\right), R / R p_{n} .
$$

These factors are isomorphic to

$$
R / R p_{1}, R / R p_{2}, \ldots, R / R p_{n-1}, R / R p_{n},
$$

respectively, because $R c / R(b c)$ is isomorphic to $R / R b$ as left modules for all nonzero elements $b, c \in R$, which can be proved by observing that the left module homomorphism $\phi_{c}$ : $R \rightarrow R / R(b c)$ given by $1 \mapsto c+R(b c)$ has kernel $R b$, and maps $R$ to $R c / R(b c)$.

An easy consequence of the length formula (2) is

Lemma 2.3 Let $a, b$ and $u$ be nonzero elements in a PID $R$. If $R(a u)=R b \cap R u$ and length $(a)=$ length $(b)$, then $R b+R u=R$.

Proof. Assume that $R b+R u=R d$ for some $d \in R$. It suffices to show length $(d)=0$. By $(2)$, length $(b u)=$ length $(b)+$ length $(u)=$ length $(d)+$ length $(a u)$, which, together with length $(a)=$ length $(b)$, implies that length $(d)=0$. 


\section{Completely reducible elements}

A nonzero element $a$ in $R$ is said to be completely reducible if it is a least common left multiple of irreducible elements in $R$, or, equivalently, the left module $R / R a$ is isomorphic to a direct sum of finitely many irreducible submodules. In particular, every irreducible element is completely reducible. The irreducible factors of a completely reducible element behave like those of a square-free element in a commutative PID.

Lemma 3.1 Let a be a completely reducible elements in a PID R. If

$$
R a=R p_{1} \cap \cdots \cap R p_{s}=R\left(q_{1} \cdots q_{t}\right),
$$

where $p_{1}, \ldots, p_{s}$ and $q_{1}, \ldots q_{t}$ are irreducible elements in $R$, and $s$ is minimal, then $s=t$ and the $p_{i}$ and $q_{j}$ are similar in pairs.

Proof. The left ideals $R p_{i}$ and $R p_{1} \cap \cdots \cap R p_{i-1} \cap R p_{i+1} \cap \cdots \cap R p_{s}$ are co-maximal for every $i$ with $1 \leq i \leq s$, because $s$ is minimal. It follows from a standard argument using the Chinese remainder theorem, that $R / R a$ is isomorphic to the direct sum $\oplus_{i=1}^{s} R / R p_{i}$, which has a composition series:

$$
0 \subset R / R p_{1} \subset R / R p_{1} \oplus R / R p_{2} \subset \cdots \subset \oplus_{i=1}^{s-1} R / R p_{i} \subset \oplus_{i=1}^{s} R / R p_{i} .
$$

From the irreducible decomposition of $a$, we have a composition series:

$$
0 \subset R\left(q_{2} \cdots q_{t}\right) / R a \subset \cdots \subset R\left(q_{t-1} q_{t}\right) / R a \subset R q_{t} / R a \subset R / R a .
$$

Therefore, $s=t$. Note that the factors of the first composition series are

$$
R / R p_{1}, \ldots, R / R p_{s}
$$

while those of the second are isomorphic to

$$
R / R q_{1}, R / R q_{2}, \ldots, R / R q_{s-1}, R / R q_{s}
$$

respectively. The $p_{i}$ and $q_{j}$ are similar in pairs by the Jordan-Hölder Theorem.

The next proposition enables us to determine the similarity of two completely reducible elements by their irreducible decompositions.

Proposition 3.2 Let $a$ and $b$ be nonzero elements of a PID $R$. Assume that $a=p_{1} \cdots p_{s}$ and $b=q_{1} \cdots q_{t}$, where the $p_{i}$ and $q_{j}$ are irreducible. If $a$ and $b$ are similar, then $s=t$ and the $p_{i}$ and $q_{j}$ are similar in pairs. The converse holds, provided that both a and $b$ are completely reducible.

Proof. If $a$ and $b$ are similar, then the two irreducible decompositions induce two equivalent composition series. Therefore, $s=t$ and the $p_{i}$ and $q_{j}$ are similar in pairs.

Assume that both $a$ and $b$ are completely reducible. If $s=t$ and the $p_{i}$ and $q_{j}$ are similar in pairs, then, by Lemma 3.1, there exist irreducible elements $p_{1}^{\prime}, \ldots, p_{s}^{\prime}$, and $q_{1}^{\prime}, \ldots, q_{s}^{\prime}$ such that

- $R / R a$ and $R / R b$ are isomorphic to $\oplus_{i=1}^{s} R / R p_{i}^{\prime}$ and $\oplus_{i=1}^{s} R / R q_{i}^{\prime}$, respectively; 
- $p_{i} \sim p_{i}^{\prime}$ and $q_{i} \sim q_{i}^{\prime}$ for all $i$ with $1 \leq i \leq s$.

Therefore, $a$ and $b$ are similar.

To study the similarity among length-two elements, we need to determine whether a product of two irreducible elements is completely reducible. The following proposition is a generalization of the mixed-equation-based criterion on whether a differential operator is a lclm of two differential operators whose gcrd is trivial (see [4]).

Proposition 3.3 Let $a, b$ be two nonzero elements in a PID $R$. There exists a nonzero element $c$ in $R$ such that

$$
R(b a)=R a \cap R c \quad \text { and } \quad R a+R c=R
$$

if and only if there exists a nonzero element $f$ in $R$ such that af -1 is in $R b$.

Proof. Assume that there exists a nonzero element $c$ in $R$ such that (3) holds. Then the map $\phi_{a}: R / R b \rightarrow R / R c$ given by $1+R b \mapsto a+R c$ is a well-defined left module isomorphism by Lemma 2.1. Assume that $\phi_{a}^{-1}$ is given by $1+R c \mapsto f+R b$. Then

$$
1+R b=\phi_{a}^{-1} \circ \phi_{a}(1+R b)=\phi_{a}^{-1}(a+R c)=a f+R b .
$$

Thus, af -1 is in $R b$.

Conversely, assume that there exists a nonzero element $f$ in $R$ such that $a f-1$ is in $R b$. Then the left module homomorphism $\psi: R \rightarrow R / R b$ given by $1 \mapsto f+R b$ is surjective, because $\psi(a)=1+R b$. Its kernel $\operatorname{ker}(\psi)$ is not 0, since $b a \in \operatorname{ker}(\psi)$. Let $c$ be a generator of $\operatorname{ker}(\psi)$. Then the induced homomorphism $\bar{\psi}: R / R c \rightarrow R / R b$ given by $1+R c \mapsto f+R b$ is an isomorphism, that is, $c$ is similar to $b$. Since

$$
\bar{\psi}(a+R c)=1+R b,
$$

the isomorphism $\bar{\psi}^{-1}: R / R b \rightarrow R / R c$ is given by $1+R b \mapsto a+R c$. So (3) holds by the remark before Lemma 2.1 .

\section{Non-completely reducible elements}

Recall that, for a nonzero element $a$ in $R$, the maximal completely reducible (right-hand) divisor of $a$ is defined to be the $\operatorname{lclm} m$ of all the right-hand irreducible divisors of $a$. In other words, $R m / R a$ is the intersection of all maximal left submodules in $R / R a$, that is, $R m / R a$ is the Jacobson radical of $R / R a$ (see [1, page 173]).

The next proposition describes a relation between two similar reducible elements and their factors.

Proposition 4.1 Let $R$ be a PID, and $p_{1}, p_{2}, q_{1}$ and $q_{2}$ be nonzero and nonunit elements in $R$. Suppose that length $\left(p_{1}\right)=$ length $\left(p_{2}\right)$ and length $\left(q_{1}\right)=\operatorname{length}\left(q_{2}\right)$. Then

(i) If there exist nonzero elements $u$ and $v$ in $R$ such that

$$
R\left(p_{1} u\right)=R p_{2} \cap R u, \quad p_{1} u=v p_{2} \quad \text { and } \quad R\left(q_{1} v\right)=R q_{2} \cap R v,
$$

then $q_{1} p_{1}$ and $q_{2} p_{2}$ are similar. 
(ii) If $q_{1} p_{1}$ and $q_{2} p_{2}$ are similar, and $p_{1}, p_{2}$ are maximal completely reducible divisors of $q_{1} p_{1}, q_{2} p_{2}$, respectively. Then the three equalities in (4) hold. In addition, we have $p_{1} \sim p_{2}$, and $q_{1} \sim q_{2}$.

Proof. Assume that there exist nonzero $u$ and $v$ in $R$ such that the three equalities in (4) hold. By Lemma 2.1, it suffices to show that

$$
R\left(q_{1} p_{1} u\right)=R\left(q_{2} p_{2}\right) \cap R u \quad \text { and } \quad R\left(q_{2} p_{2}\right)+R u=R .
$$

We compute

$$
\begin{aligned}
R\left(q_{2} p_{2}\right) \cap R u & =R\left(q_{2} p_{2}\right) \cap R u \cap R p_{2} \\
& =R\left(q_{2} p_{2}\right) \cap R\left(p_{1} u\right) \quad \text { (by the first equality in (4)) } \\
& =R\left(q_{2} p_{2}\right) \cap R\left(v p_{2}\right) \quad \text { (by the second equality in (4)) } \\
& =R\left(q_{1} v p_{2}\right) \quad \text { (by the third equality in (4)) } \\
& =R\left(q_{1} p_{1} u\right) \quad \text { (by the first equality in (4)). }
\end{aligned}
$$

The first equality in (5) holds. This equality and Lemma 2.3 yield the second in (5).

We now assume that $p_{1}$ and $p_{2}$ are maximal completely reducible divisors of $q_{1} p_{1}$ and $q_{2} p_{2}$, respectively. Put $I_{1}=R\left(q_{1} p_{1}\right)$ and $I_{2}=R\left(q_{2} p_{2}\right)$. If $q_{1} p_{1} \sim q_{2} p_{2}$, then there is an isomorphism $\phi$ from $R / I_{1}$ to $R / I_{2}$. Assume that $\phi\left(1+I_{1}\right)=u+I_{2}$ with $u \in R$. Then the two equalities in (5) hold by Lemma 2.1. Note that $R p_{1} / I_{1}$ and $R p_{2} / I_{2}$ are the Jacobson radicals of $R / I_{1}$ and $R / I_{2}$, respectively. So $\phi\left(R p_{1} / I_{1}\right)=R p_{2} / I_{2}$ ([1, Proposition 4.3.6]), which induces an isomorphism from $\left(R / I_{1}\right) /\left(R p_{1} / I_{1}\right)$ to $\left(R / I_{2}\right) /\left(R p_{2} / I_{2}\right)$. As a direct consequence of the third isomorphism theorem, there is an isomorphism from $R / R p_{1}$ to $R / R p_{2}$ that maps $1+R p_{1}$ to $u+R p_{2}$. Hence, $R\left(p_{1} u\right)=R p_{2} \cap R u$ by Lemma 2.1. Let $v$ be the element in $R$ such that $p_{1} u=v p_{2}$. It remains to verify the third equality in (4). We compute

$$
\begin{aligned}
R\left(q_{1} v p_{2}\right) & =R\left(q_{1} p_{1} u\right) \quad(\text { by the second equality in }(4)) \\
& =R\left(q_{2} p_{2}\right) \cap R u \quad \text { (by the first equality in (5)). } \\
& =R\left(q_{2} p_{2}\right) \cap R u \cap R p_{2} \\
& =R\left(q_{2} p_{2}\right) \cap R\left(p_{1} u\right) \quad \text { (by the first equality in (4)) } \\
& =R\left(q_{2} p_{2}\right) \cap R\left(v p_{2}\right) \quad \text { (by the second equality in (4)). }
\end{aligned}
$$

Hence, $R\left(q_{1} v p_{2}\right)=R\left(q_{2} p_{2}\right) \cap R\left(v p_{2}\right)$. Removing $p_{2}$ yields the third equality in (4).

From the first and third equalities in (4) and Lemma 2.3 it follows that

$$
R p_{2}+R u=R=R q_{2}+R v .
$$

The last assertion holds by Lemma 2.1.

In practice, it is not easy to compute the Jacobson radical of a left module. However, Proposition 4.1 can be applied to the following simple situation. Let $p_{1}, p_{2}, q_{1}, q_{2}$ be irreducible elements in a PID $R$. To determine whether $q_{1} p_{1}$ and $q_{2} p_{2}$ are similar, we make the following case distinction:

1. If one of the $q_{i} p_{i}$ is completely reducible, while the other is not, then they are not similar, because their corresponding modules are not isomorphic. 
2. If both of them are completely reducible, then they are similar if and only if the factors are similar in pairs by Proposition 3.2.

3. If neither of them is completely reducible, then we use the next conclusion.

Proposition 4.2 Let $R$ be a PID, $p_{1}, p_{2}, q_{1}$ and $q_{2} \in R$ be irreducible. Suppose that neither $q_{1} p_{1}$ nor $q_{2} p_{2}$ is completely reducible. Then $q_{1} p_{1} \sim q_{2} p_{2}$ if and only if the three equalities in (4) hold.

Proof. According to Proposition 4.1, it suffices to show that $p_{1}$ (resp. $p_{2}$ ) is the maximal completely reducible divisor of $q_{1} p_{1}$ (resp. $q_{2} p_{2}$ ). Assume that $p_{1}^{\prime}$ is an irreducible right-hand divisor of $q_{1} p_{1}$ with $R p_{1}^{\prime} \neq R p_{1}$. Then the $\operatorname{lclm} s$ of $p_{1}$ and $p_{1}^{\prime}$ has length two by Lemma 3.1, and $s$ is a right-hand divisor of $q_{1} p_{1}$ by definition. Since length $\left(q_{1} p_{1}\right)=2$,

$$
R\left(q_{1} p_{1}\right)=R s=R p_{1} \cap R p_{1}^{\prime} .
$$

The element $q_{1} p_{1}$ is completely reducible, a contradiction. Hence, $p_{1}$ is the maximal completely reducible divisor of $q_{1} p_{1}$. The same conclusion holds for $p_{2}$.

Let $q_{1}, p_{1}, q_{2}, p_{2}$ be given in Proposition 4.2. To decide whether $q_{1} p_{1} \sim q_{2} p_{2}$, we first decide whether $p_{1} \sim p_{2}$ and $q_{1} \sim q_{2}$. If either does not hold, $q_{1} p_{1} \nsim q_{2} p_{2}$. Otherwise, there exists a pair $(u, v)$ with $u v \neq 0$ such that

$$
R\left(p_{1} u\right)=R p_{2} \cap R u \quad \text { and } \quad R\left(q_{1} v\right)=R p_{2} \cap R v
$$

by Lemma 2.1. There are many such pairs, some of which are described in the next corollary.

Corollary 4.3 Let $p_{1}, p_{2}, q_{1}$ and $q_{2}$ be the same as those in Proposition 4.2. If the three equalities in (4) hold, then, for any $\hat{u} \in R$ such that $\hat{u}-u \in R\left(q_{2} p_{2}\right)$, there exists $\hat{v} \in R$ such that

$$
R\left(p_{1} \hat{u}\right)=R p_{2} \cap R \hat{u}, \quad p_{1} \hat{u}=\hat{v} p_{2} \quad \text { and } \quad R\left(q_{1} \hat{v}\right)=R q_{2} \cap R \hat{v} .
$$

Proof. By the first part in the proof of Proposition 4.1, we have that

$$
R\left(q_{1} p_{1} u\right)=R\left(q_{2} p_{2}\right) \cap R u \quad \text { and } \quad R\left(q_{2} p_{2}\right)+R u=R .
$$

Then, for every $\hat{u} \in R$ with $\hat{u}-u \in R\left(q_{2} p_{2}\right)$,

$$
R\left(q_{1} p_{1} \hat{u}\right)=R\left(q_{2} p_{2}\right) \cap R \hat{u} \quad \text { and } \quad R\left(q_{2} p_{2}\right)+R \hat{u}=R
$$

by Lemma 2.1. Hence, there exists $\hat{v} \in R$ such that the three equalities in (6) hold by the second part of the proof of Proposition 4.1.

\section{Applications to univariate Ore polynomial rings}

In this section, we apply the results obtained in Sections 3. and 4. to determine whether two reducible quadratic Ore polynomials are similar, assuming that their irreducible decompositions are given. Such a situation arises when one classifies second-order right-hand divisors of a differential (difference) operator with respect to similarity. 
Let $k$ be a commutative field, $\sigma$ an automorphism of $k$, and $\delta$ a derivation on $k$ with respect to $\sigma$, that is, $\delta$ is additive and

$$
\delta\left(r_{1} r_{2}\right)=\sigma\left(r_{1}\right) \delta\left(r_{2}\right)+\delta\left(r_{1}\right) r_{2} \quad \text { for all } r_{1}, r_{2} \in k .
$$

A univariate Ore polynomial ring $k[\partial ; \sigma, \delta]$ over $k$ is the ring of polynomials in $\partial$ over $k$ with the commutation rule $\partial r=\sigma(r) \partial+\delta(r)$ for any $r \in k$. For example, $\mathbb{Q}(x)[\partial ; \mathbf{1}, \delta]$, where $\mathbf{1}$ maps each element to itself, and $\delta=\frac{\mathrm{d}}{\mathrm{d} x}$, is the ring of differential operators over $\mathbb{Q}(x)$; and $\mathbb{Q}(n)[\partial ; \sigma, \mathbf{0}]$, where $\sigma$ maps $f(n)$ to $f(n+1)$ for all $f(n) \in \mathbb{Q}(n)$, and $\mathbf{0}$ maps everything to zero, is the ring of shift operators over $\mathbb{Q}(n)$. The ring $k[\partial ; \sigma, \delta]$ possesses the right and left division algorithms, hence it is a PID $([7,6])$.

The following lemma is immediate from [6, page 15]. Our proof is an easy application of Lemma 2.1.

Lemma 5.1 Two degree-one Ore polynomials $\partial-a$ and $\partial-b$ in $k[\partial ; \sigma, \delta]$ are similar if and only if there exists nonzero element $u \in k$ such that

$$
\delta(u)+b \sigma(u)-a u=0 .
$$

Proof. Put $A=\partial-a, B=\partial-b$ and $R=k[\partial ; \sigma, \delta]$. By Lemma 2.1, $A \sim B$ if and only if there exists a nonzero element $u \in k$ such that $R(A u)=R B \cap R u$, which equals $R B$. It is straightforward to verify that $R(A u)=R B$ if and only if $u$ satisfies (7).

When $\sigma$ is the identity map, (7) says that $\partial-a$ and $\partial-b$ are similar if and only if $a-b$ is a logarithmic derivative. When $\delta$ maps everything to zero, the two operators are similar if and only if either $a=b=0$ or $a / b=\sigma(u) / u$. The former coincides with the similarity of exponential functions, while the latter with hypergeometric terms.

The next lemma tells us how to determine whether a product of two degree-one Ore polynomials is completely reducible.

Lemma 5.2 Let $A=\partial-a, B=\partial-b$ be two operators in $k[\partial ; \sigma, \delta]$. Then $B A$ is completely reducible if and only if the equation

$$
\delta(z)+b \sigma(z)-a z=1
$$

has a solution in $k$. Moreover, $B A=\operatorname{lclm}\left(A, A-\frac{1}{u}\right)$, provided that $u \in k$ solves (8).

Proof. By Proposition 3.3, $B A$ is completely reducible if and only if there exists $r$ in $k[\partial ; \sigma, \delta]$ such that $A r \equiv 1 \bmod B$. Clearly, the right-hand remainder of $r$ by $B$ also satisfies the above congruence when $r$ does. Hence, we may assume further that $r$ belongs to $k$. Expanding $A r$ yields (8). The first part of the lemma follows. The last assertion follows from the fact that the right-hand remainder of $B A$ by $A-\frac{1}{u}$ is zero if and only if $u \in k$ is a solution of (8).

Example 5.3 If $\partial^{2}$ is considered as a differential operator in $\mathbb{Q}(x)\left[\partial ; \mathbf{1}, \frac{\mathrm{d}}{\mathrm{d} x}\right]$, then (8) has a solution $x$. So $\partial^{2}$ is completely reducible and $\partial^{2}=\operatorname{lclm}\left(\partial, \partial-\frac{1}{x}\right)$. If $\partial^{2}$ is regarded as an element in $k[\partial ; \sigma, \mathbf{0}]$, where $\sigma$ is an automorphism on $k$, then (8) does not hold. So $\partial^{2}$ is not completely reducible in $k[\partial ; \sigma, \mathbf{0}]$. 
Lemma 5.2 yields the following algorithm.

Algorithm IsCompletelyReducible. Given two elements $a, b$ in a differential field $(k, \delta)$, decide whether $(\partial-b)(\partial-a)$ is completely reducible in $k[\partial ; \mathbf{1}, \delta]$.

1. Determine whether the equation

$$
\delta(z)+(b-a) z=1
$$

has a solution in $k$.

2. If there is a solution, then return true $[(\partial-b)(\partial-a)$ is completely reducible]. Otherwise, false is returned.

One can determine whether the product of two first-order difference operators is completely reducible in a similar manner.

The main result of this section is given in the next proposition.

Proposition 5.4 Let $A_{i}=\partial-a_{i}$ and $B_{i}=\partial-b_{i}$ be in $k[\partial ; \sigma, \delta]$ for $i=1,2$. If neither $B_{1} A_{1}$ nor $B_{2} A_{2}$ is completely reducible, then $B_{1} A_{1}$ is similar to $B_{2} A_{2}$ if and only if there exist $u$, $v$ and $w$ in $k$ with $u v \neq 0$ such that

$$
\begin{aligned}
\delta(u)+a_{2} \sigma(u)-a_{1} u & =0 \\
\delta(v)+b_{2} \sigma(v)-b_{1} v & =0 \\
\delta(w)+b_{2} \sigma(w)-a_{1} w+\sigma(u)-v & =0 .
\end{aligned}
$$

Proof. Put $R=k[\partial ; \sigma, \delta]$. Assume that $B_{1} A_{1} \sim B_{2} A_{2}$. By Proposition 4.2, there exist nonzero elements $U$ and $V$ in $k[\partial ; \sigma, \delta]$ such that

$$
R\left(A_{1} U\right)=R A_{2} \cap R U, A_{1} U=V A_{2}, R\left(B_{1} V\right)=R B_{2} \cap R V .
$$

We may further assume that $U$ and $V$ are of degrees at most one by Corollary 4.3. The first equality in (12) implies that $R A_{2}+R U=R$ by Lemma 2.3 .

Likewise, the third equality implies that $R B_{2}+R V=R$. So $U$ and $V$ can be written, respectively, as

$$
U=w A_{2}+u \quad \text { and } \quad V=\sigma(w) B_{2}+v, \quad \text { where } u, v, w \in k \text { and } u v \neq 0,
$$

Note that the leading coefficient of $V$ is equal to $\sigma(w)$ because of the second equality in (12). Consequently, $R\left(A_{1} u\right)=R A_{2} \cap R u=R A_{2}$ by the first equality in (12) and the last assertion of Lemma 2.1. Therefore, we have

$$
A_{1} u=\sigma(u) A_{2}
$$

Expanding (14) yields (9). The third equality in (12) implies (10) in the same vein. To verify (11), we observe that $A_{1} U=\left(A_{1} w+\sigma(u)\right) A_{2}$ by (14). Hence, the second equality in (12) implies $V=A_{1} w+\sigma(u)$, which, together with the definition of $V$ given in (13), implies that $A_{1} w+\sigma(u)=\sigma(w) B_{2}+v$. Equation (11) follows at once. 
Conversely, suppose that there exist $u, v$ and $w$ in $k$ with $u v \neq 0$ such that (9), (10) and (11) hold. Then (14) holds by (9). Consequently, $R\left(A_{1} u\right)=R A_{2} \cap R u$. In the same vein, $R\left(B_{1} v\right)=R B_{2} \cap R v$ by (10). Let

$$
U=w A_{2}+u \quad \text { and } \quad V=\sigma(w) B_{2}+v .
$$

Then the first and third equalities in (12) hold by the last assertion of Lemma 2.1. Furthermore, (11) implies that the second equality in (12) holds. Thus, $B_{1} A_{1} \sim B_{2} A_{2}$ by Proposition 4.2.

Corollary 5.5 Let $A_{i}=\partial-a_{i}$ and $B_{i}=\partial-b_{i}$ be in $k[\partial ; \mathbf{1}, \delta]$ for $i=1,2$. If neither $B_{1} A_{1}$ nor $B_{2} A_{2}$ is completely reducible, then $B_{1} A_{1} \sim B_{2} A_{2}$ if and only if there exist $u, v$ and $w$ in $k$ with $u v \neq 0$ such that

$$
\begin{aligned}
\delta(u)+\left(a_{2}-a_{1}\right) u & =0 \\
\delta(v)+\left(b_{2}-b_{1}\right) v & =0 \\
\delta(w)+\left(b_{2}-a_{1}\right) w+u-v & =0 .
\end{aligned}
$$

Note that, in the differential case, if $L_{1}=\partial^{2}+f_{1} \partial+f_{2}, L_{2}=\partial^{2}+g_{1} \partial+g_{2}$, the mixed equation $L_{1} U \equiv 0 \bmod L_{2}$ leads to the following system of differential equations

$$
\left\{\begin{aligned}
\delta^{2}\left(u_{1}\right)+\left(f_{1}-2 g_{1}\right) \delta\left(u_{1}\right)+2 \delta\left(u_{2}\right) & =F_{1} u_{1}+\left(g_{1}-f_{1}\right) u_{2} \\
\delta^{2}\left(u_{2}\right)-2 g_{2} \delta\left(u_{1}\right)+f_{2} \delta\left(u_{2}\right) & =F_{2} u_{1}+\left(g_{2}-f_{2}\right) u_{2},
\end{aligned}\right.
$$

where $U=u_{1} \partial+u_{2}, \quad F_{1}=\delta\left(g_{1}\right)+f_{1} g_{1}-g_{1}^{2}-f_{2}+g_{2}$ and $F_{2}=\delta\left(g_{2}\right)+f_{1} g_{2}-g_{1} g_{2}$. Corollary 5.5 takes advantage of the factors to decouples this system into two first-order scalar differential equations, and a Risch equation in $w$. If both (15) and (16) have nonzero solutions in $k$, we need to solve the parametric Risch equation:

$$
\delta(w)+\left(b_{2}-a_{1}\right) w=c_{2} v-c_{1} u
$$

for $w$ and unspecified nonzero constants $c_{1}$ and $c_{2}$ in $k$. Note that the parametric Risch equation has been studied intensively due to its fundamental importance in symbolic integration (see, $[2$, Chapter 7] and the references therein).

Corollary 5.6 Let $A_{i}=\partial-a_{i}$ and $B_{i}=\partial-b_{i}$ be in $k[\partial ; \sigma, \mathbf{0}]$ for $i=1,2$. If neither $B_{1} A_{1}$ nor $B_{2} A_{2}$ is completely reducible, then $B_{1} A_{1} \sim B_{2} A_{2}$ if and only if there exist $u, v$ and $w$ in $k$ with $u v \neq 0$ such that

$$
\begin{aligned}
a_{2} \sigma(u)-a_{1} u & =0 \\
b_{2} \sigma(v)-b_{1} v & =0 \\
b_{2} \sigma(w)-a_{1} w+\sigma(u)-v & =0 .
\end{aligned}
$$

Equation (19) is the difference analog of Risch's equation. An algorithm for computing solutions of (19) in $k$ can be found in [9].

Under the assumption that the solutions of parametric Risch's equation in $k$ are computable, we present an algorithm for determine whether $B_{1} A_{1}$ and $B_{2} A_{2}$ are similar in the differential case. 
Algorithm IsSimilar. Given $a_{1}, a_{2}, b_{1}, b_{2}$ in a differential field $(k, \delta)$, determine whether

$$
\left(\partial-b_{1}\right)\left(\partial-a_{1}\right) \sim\left(\partial-b_{2}\right)\left(\partial-a_{2}\right),
$$

where the product is taken in the ring $k[\partial ; \mathbf{1}, \delta]$.

[We set $L_{1}=\left(\partial-b_{1}\right)\left(\partial-a_{1}\right)$ and $L_{2}=\left(\partial-b_{2}\right)\left(\partial-a_{2}\right)$ for brevity.]

1. Determine whether $L_{1}$ and $L_{2}$ are completely reducible by the algorithm IsCompetelyReducible.

2. If one of them is completely reducible but the other is not, then return false $\left[L_{1}\right.$ and $L_{2}$ are not similar].

3. If both $L_{1}$ and $L_{2}$ are completely reducible, then do the following.

3.1. If both $\delta(z)+\left(a_{2}-a_{1}\right) z=0$ and $\delta(z)+\left(b_{2}-b_{1}\right) z=0$ have nonzero solutions in $k$, then return true [ $L_{1}$ and $L_{2}$ are similar].

3.2. If both $\delta(z)+\left(b_{2}-a_{1}\right) z=0$ and $\delta(z)+\left(a_{2}-b_{1}\right) z=0$ have nonzero solutions in $k$, then return true $\left[L_{1}\right.$ and $L_{2}$ are similar].

3.3. Return false.

4. If neither $L_{1}$ nor $L_{2}$ is completely reducible, then do the following

4.1. Determine if the equation

$$
\delta(z)+\left(a_{2}-a_{1}\right) z=0
$$

has a nonzero solution in $k$. If there is none, then return false $\left[L_{1}\right.$ and $L_{2}$ are not similar]. Otherwise, set $u$ to be a solution of the above equation.

4.2. Determine if the equation

$$
\delta(z)+\left(b_{2}-b_{1}\right) z=0
$$

has a nonzero solution in $k$. If there is none, then return false $\left[L_{1}\right.$ and $L_{2}$ are not similar]. Otherwise, set $v$ to be a solution of the above equation.

4.3. Determine if there exists $w \in k$ and nonzero constants $c_{1}, c_{2}$ in $k$ such that

$$
\delta(w)+\left(b_{2}-a_{1}\right) w+c_{1} u-c_{2} v=0 .
$$

If there exist such $w, c_{1}$ and $c_{2}$, then return true $\left[L_{1}\right.$ and $L_{2}$ are not similar]. Otherwise false is returned.

The second step of this algorithm is obviously correct. The third step is correct by Proposition 3.2, and the fourth by Corollary 5.5. We remark that this algorithm can be modified to get an operator $U \in k[\partial ; \mathbf{1}, \delta]$ such that $L_{1} U=\operatorname{lclm}\left(L_{2}, U\right)$ if $L_{1} \sim L_{2}$.

Similarly, Corollary 5.6 leads to an algorithm for determining whether two difference operators $\left(\partial-b_{1}\right)\left(\partial-a_{1}\right)$ and $\left(\partial-b_{2}\right)\left(\partial-a_{2}\right)$ in $k[\partial ; \sigma, \mathbf{0}]$ are similar. But some special care should be taken when $a_{1}=a_{2}=0$ or $b_{1}=b_{2}=0$ in the difference case. 


\section{Examples}

We present a few examples illustrating the methods described in Section 5.. In some examples, we analyze the behavior of the right-hand divisors of Ore polynomials with respect to similarity; in the others, we compare our method with the method based on mixed equations.

The first example shows that two differential operators may be not similar even if their factors are similar in pairs.

Example 6.1 $R=\mathbb{Q}(x)\left[\partial ; \mathbf{1}, \frac{\mathrm{d}}{\mathrm{d} x}\right], A=\partial-x^{2}, B_{1}=\partial-\frac{1}{x+1}, B_{2}=\partial-\frac{1}{x}$. By the algorithm Is CompletelyReducible, neither $B_{1} A$ nor $B_{2} A$ is completely reducible. Solving the equations

$$
\frac{\mathrm{d} u}{\mathrm{~d} x}=0 \quad \text { and } \quad \frac{\mathrm{d} v}{\mathrm{~d} x}+\left(\frac{1}{x}-\frac{1}{x+1}\right) v=0
$$

in $\mathbb{Q}(x)$ yields $u=1$ and $v=\frac{x+1}{x}$, which implies that $B_{1} \sim B_{2}$. But the equation

$$
\frac{\mathrm{d} w}{\mathrm{~d} x}+\left(\frac{1}{x}-x^{2}\right) w+c_{1}-c_{2} \frac{x+1}{x}=0
$$

has no solution $w$ in $\mathbb{Q}(x)$ for any $c_{1}, c_{2}$ in $\mathbb{Q} \backslash\{0\}$. So $B_{1} A$ and $B_{2} A$ are not similar.

A differential operator may have infinitely many second-order right-hand divisors that are dissimilar to each other, as given in the next example.

Example 6.2 Let $R$ and $A$ be the same as Example 6.1, and let $L=\partial^{2} A$. It is easy to verify that $\left(\partial-\frac{1}{x+c}\right) A$ is a right-hand divisor of $L$ for every $c \in \mathbb{Q}$. These divisors are not completely reducible, because

$$
\frac{\mathrm{d} z}{\mathrm{~d} x}+\left(\frac{1}{x+c}-x^{2}\right) z=1
$$

has no solution in $\mathbb{Q}(x)$. We show further that these divisors are dissimilar to each other. Put $P_{c}=\left(\partial-\frac{1}{x+c}\right) A$ and $P_{d}=\left(\partial-\frac{1}{x+d}\right) A$ with $c, d \in \mathbb{Q}$. Solving the equations

$$
\frac{\mathrm{d} u}{\mathrm{~d} x}=0 \quad \text { and } \quad \frac{\mathrm{d} v}{\mathrm{~d} x}+\left(\frac{1}{x+d}-\frac{1}{x+c}\right) v=0
$$

in $\mathbb{Q}(x)$ yields $u=1$ and $v=\frac{x+c}{x+d}$. Then $P_{c} \sim P_{d}$ if and only if the following equation

$$
\frac{\mathrm{d} w}{\mathrm{~d} x}+\left(\frac{1}{x+d}-x^{2}\right) w+c_{1}-c_{2} \frac{x+c}{x+d}=0
$$

has a solution $w$ in $\mathbb{Q}(x)$ for some $c_{1}, c_{2}$ in $\mathbb{Q} \backslash\{0\}$. Substituting $w=\frac{y}{x+d}$ in (20) yields

$$
\frac{\mathrm{d} y}{\mathrm{~d} x}-x^{2} y+\left(c_{1}-c_{2}\right) x+c_{1} d-c_{2} c=0,
$$

which has no nonzero solution in $\mathbb{Q}(x)$. If $y=0$, then $c_{1}=c_{2}$ and, hence, $c=d$. Thus, $P_{c} \sim$ $P_{d}$ if and only if $P_{c}=P_{d}$. 
In the next two examples, we compare our algorithm with a naive use of the method based on mixed equations.

Example 6.3 Let $R=\mathbb{Q}(x)\left[\partial ; \mathbf{1}, \frac{\mathrm{d}}{\mathrm{d} x}\right], A_{1}=\partial, A_{2}=\partial+\frac{1}{x}$, and $B=\partial-x$. By the algorithm Is CompletelyReducible, $B A_{2}$ is completely reducible while $B A_{1}$ is not. Hence $B A_{1}$ and $B A_{2}$ are not similar.

If we solve the mixed equation

$$
\left(B_{1} A_{1}\right) U \equiv 0 \bmod B_{2} A_{2}
$$

by the function Homomorphisms in the Maple package DEtools, which is of form as (18), we get all the homomorphism $\phi_{c}$ from $R / R\left(B_{1} A_{1}\right)$ to $R / R\left(B_{2} A_{2}\right)$ given by

$$
1+R\left(B_{1} A_{1}\right) \mapsto \underbrace{c\left(\partial-\frac{x^{2}-1}{x}\right)}_{L_{c}}+R\left(B_{2} A_{2}\right),
$$

where $c$ is an arbitrary element in $\mathbb{Q}$. For any nonzero $c \in \mathbb{Q}, \phi_{c}$ is not an isomorphism, Since $R L_{c}+R\left(B_{2} A_{2}\right)=R\left(\partial-\frac{x^{2}-1}{x}\right)$. Consequently, $B_{1} A_{1}$ and $B_{2} A_{2}$ are not similar.

Example 6.4 Let $R=\mathbb{Q}(x)\left[\partial ; \mathbf{1}, \frac{\mathrm{d}}{\mathrm{d} x}\right]$,

$$
A_{1}=\partial+\frac{1}{x}, B_{1}=\partial-\frac{1}{x(x+1)}, A_{2}=\partial, B_{2}=\partial+\frac{1}{x+1} \in R .
$$

Neither $B_{1} A_{1}$ nor $B_{2} A_{2}$ is completely reducible. Solving the equations

$$
\frac{\mathrm{d} u}{\mathrm{~d} x}+\frac{1}{x} u=0 \quad \text { and } \quad \frac{\mathrm{d} v}{\mathrm{~d} x}-\frac{1}{x} v=0
$$

in $\mathbb{Q}(x)$, we obtain a solution $u=\frac{1}{x}$ and $v=x$, respectively. Next, we decide if

$$
\frac{\mathrm{d} w}{\mathrm{~d} x}+\frac{1}{x(x+1)} w+c_{1} \frac{1}{x}-c_{2} x=0
$$

has a solution $w$ in $\mathbb{Q}(x)$ for some $c_{1}, c_{2}$ in $\mathbb{Q} \backslash\{0\}$. Indeed, it has a solution

$$
\left(c_{1}, c_{2}, w\right)=\left(2,2, x^{2}-x-2\right) .
$$

Hence, $B_{1} A_{1} \sim B_{2} A_{2}$.

If we solve the mixed equation

$$
\left(B_{1} A_{1}\right) U \equiv 0 \quad \bmod B_{2} A_{2}
$$

by the function Homomorphisms in the Maple package DEtools, which is of form as (18), we get all the homomorphism $\phi_{d_{1}, d_{2}}$ from $R / R\left(B_{1} A_{1}\right)$ to $R / R\left(B_{2} A_{2}\right)$ given by the rule

$$
1+R\left(B_{1} A_{1}\right) \mapsto \underbrace{d_{1} \frac{(x+1) \partial}{x}+d_{2}\left((x+1)(x-2) \partial+\frac{2}{x}\right)}_{L_{d_{1}, d_{2}}}+R\left(B_{2} A_{2}\right)
$$


where $d_{1}$ and $d_{2}$ are arbitrary elements in $\mathbb{Q}$. We need to decide if there exist $d_{1}$ and $d_{2}$ in $\mathbb{Q}$ such that $\phi_{d_{1}, d_{2}}$ is an isomorphism. Indeed, it is an isomorphism if and only if the gcrd of $B_{2} A_{2}$ and $L_{d_{1}, d_{2}}$ is trivial, which can be done by a resultant-based method. We have that $\phi_{0,1}$ is an isomorphism.

The next example shows that our algorithm can be applied to operators with coefficients in a monomial extension over $\mathbb{Q}(x)$, thanks to the algorithms developed for Risch's equation $($ see $[8,2])$.

Example 6.5 Let $R=\mathbb{Q}(x, \log (x))\left[\partial ; \mathbf{1}, \frac{\mathrm{d}}{\mathrm{d} x}\right]$,

$$
A_{1}=\partial, B_{1}=\partial-\frac{\log (x)-1}{x \log (x)}, A_{2}=\partial-\frac{1-\log (x)}{x \log (x)}, B_{2}=\partial+\frac{1}{x \log (x)} \in R .
$$

Neither $B_{1} A_{1}$ nor $B_{2} A_{2}$ is completely reducible by Algorithm IsCompletelyReducible. Solving the equations

$$
\frac{\mathrm{d} u}{\mathrm{~d} x}+\frac{1-\log (x)}{x \log (x)} u=0 \quad \text { and } \quad \frac{\mathrm{d} v}{\mathrm{~d} x}-\frac{1}{x} v=0
$$

in $\mathbb{Q}(x, \log (x))$ yields $u=\frac{x}{\log (x)}$ and $v=x$, respectively. The corresponding parametric Risch's equation

$$
\frac{\mathrm{d} w}{\mathrm{~d} x}-\frac{1}{x \log (x)} w+c_{1} \frac{x}{\log (x)}-c_{2} x=0
$$

has a solution $\left(c_{1}, c_{2}, w\right)=\left(1,2, x^{2}\right)$. So $B_{1} A_{1} \sim B_{2} A_{2}$.

The next example is for difference operators.

Example 6.6 Let $R=\mathbb{Q}(n, n !)[\partial ; \sigma, \mathbf{0}]$ where $\sigma(n)=n+1$, and $\sigma(n !)=(n+1)$ !.

$$
A_{1}=\partial-\frac{(n-1) n !}{n}, B_{1}=\partial-\frac{n}{n+2}, A_{2}=\partial-n !, B_{2}=\partial-n \in R .
$$

Neither $B_{1} A_{1}$ nor $B_{2} A_{2}$ is completely reducible by Lemma 5.2. Solving the equations

$$
n ! \sigma(u)-\frac{(n-1) n !}{n} u=0 \text { and } n \sigma(v)-\frac{n}{n+2} v=0
$$

in $\mathbb{Q}(n, n !)$ yields a solution $u=\frac{1}{n-1}$ and $v=\frac{1}{(n+1) !}$, respectively. Next we decide whether the following parameterized linear difference equation

$$
n \sigma(w)-\frac{(n-1) n !}{n} w+c_{1} \frac{1}{n}-c_{2} \frac{1}{(n+1) !}=0
$$

has a solution $w$ in $\mathbb{Q}(n, n !)$ for some $c_{1}, c_{2}$ in $\mathbb{Q} \backslash\{0\}$ (see [9]). Solving this equation yields a solution $\left(c_{1}, c_{2}, w\right)=\left(1,1, \frac{1}{(n-1) n !}\right)$. So $B_{1} A_{1} \sim B_{2} A_{2}$. 


\section{Concluding remarks}

In this paper, we developed some techniques for determining similarities of Ore polynomials using their irreducible decompositions. A complete algorithm is given for reducible quadratic Ore polynomials. For cubic Ore polynomials, we need to know their maximal completely reducible (right-hand) divisors in order to apply Proposition 4.1. An interesting question is how to determine the maximal completely reducible (right-hand) divisor of a product of irreducible Ore polynomials.

\section{Acknowledgement}

We thank P. Paule and C. Schneider at RISC-Linz for allowing us to use the Mathematica package Sigma to solve parametric linear difference equations.

\section{References}

[1] A.J. Berrick and M.E. Keating. An Introduction to Rings and Modules: with K-theory in view. Cambridge University Press, 2000.

[2] M. Bronstein. Symbolic Integration I: Transcendental Functions. Springer-Verlag, 2005.

[3] M. Bronstein and M. Petkovšek. An introduction to pseudo-linear algebra. Theoretical Computer Science, 157(1), 3-33, 1996.

[4] M. van Hoeij. Rational solutions of the mixed differential equation and its application to factorization of differential operators. Proceedings ISSAC'96, ACM Press, 219-225, 1996.

[5] N. Jacobson. The Theory of Rings. American Math. Soc., New York, 1943.

[6] N. Jacobson. Finite-Dimensional Division Algebras over Fields. Springer-Verlag, 1996.

[7] O. Ore. Theory of non-commutative polynomials. Annals of Mathematics, 34(22), 480-508, 1933.

[8] R. Risch. The problem of integration in finite terms. Trans. Amer. Math. Soc., 139, 167-189, 1969.

[9] C. Schneider. Solving parameterized linear difference equations in terms of indefinite nested sums and products. J. Differ. Equations Appl., 11(9), 799-821, 2005.

[10] M.F. Singer. Testing reducibility of linear differential operators: A group theoretic perspective. Appl. Algebra Eng., Commun. Comput., 7(2), 77-104, 1996. 